\title{
Idade e crescimento de Callinectes danae e C. ornatus (Crustacea, Decapoda) na Baía de Guanabara, Rio de Janeiro, Brasil
}

\author{
Karina A. Keunecke ${ }^{1}$, Fernando D’Incao ${ }^{2}$, Francisco N. Moreira ${ }^{1}$, Demarques R. Silva Jr. ${ }^{1}$ \& \\ José R. Verani ${ }^{3}$
}

1. Universidade Federal do Rio de Janeiro, Instituto de Biologia, Depto. de Biologia Marinha, Laboratório de Biologia e Tecnologia Pesqueira, CCS-B1. A, 21949-900 Cidade Universitária, Ilha do Fundão, Rio de Janeiro, RJ, Brasil. (keunecke@biologia.ufrj.br)

2. Fundação Universidade de Rio Grande, Depto. de Oceanografia, Laboratório de Crustáceos Decápodes, Caixa Postal 474, $96201-900$ Rio Grande, RS, Brasil.

3. Universidade Federal de São Carlos, Depto. de Hidrobiologia, Laboratório de Dinâmica de Populações de Peixes, Caixa Postal 676, 13565-905 São Carlos, SP, Brasil.

\begin{abstract}
Age and growth of Callinectes danae and C. ornatus (Crustacea, Decapoda) in Guanabara Bay, Rio de Janeiro, Brazil. Callinectes danae Smith, 1869 and C. ornatus Ordway, 1863 constitute an important portion from the fishing products at Guanabara Bay. Both species compose a significant by-catch fraction being a lot discarded during shrimp trawlings along Brazilian coast. Growth curves were estimated by modal progression analysis. For the biological parameters it was applied the Bertalanffy growth function and for the age it was used its inverse formula. Growth curves of $C$. danae and $C$. ornatus were respectively: $\sigma$ LC $=$ $120\left(1-\mathrm{e}^{-0,005 t}\right), \& \mathrm{LC}=113\left(1-\mathrm{e}^{-0,005 t}\right) ; \sigma^{-7} \mathrm{LC}=94\left(1-\mathrm{e}^{-0,005 t}\right)$ and $\subsetneq \mathrm{LC}=110\left(1-\mathrm{e}^{-0,005 t}\right)$. The age for both species reached around 2.5 years, besides to confirm growth curves. Growth study in crustaceans is very important, because besides supplying biological information it subsidizes fishery management for exploted species.
\end{abstract}

KEYWORDS. Fishery, management, swimmingcrab, by-catch.

RESUMO. Callinectes danae Smith, 1869 e C. ornatus Ordway, 1863 constituem uma parcela importante da produção pesqueira na Baía de Guanabara. Ambas espécies compõem uma fração significativa da fauna-acompanhante sendo exaustivamente descartadas pelas pescarias de arrasto de camarões na costa brasileira. As curvas de crescimento de $C$. danae e $C$. ornatus foram calculadas por meio da análise de progressão modal. Para estimar os parâmetros biológicos, foi aplicado o modelo de crescimento de Bertalanffy e para

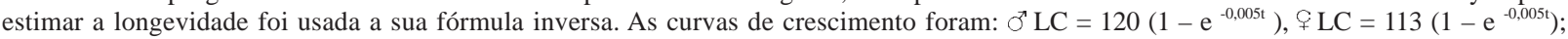
$\sigma^{T} \mathrm{LC}=94\left(1-\mathrm{e}^{-0,005 t}\right)$ e $\subseteq \mathrm{LC}=110\left(1-\mathrm{e}^{-0,005 t}\right)$, respectivamente para $C$. danae e $C$. ornatus. A longevidade alcançada para as duas espécies foi em torno de 2,5 anos, além de validar as curvas de crescimento. O estudo do crescimento em crustáceos é de extrema importância, pois além de fornecer a informação biológica básica para o grupo, os parâmetros estimados subsidiam o ordenamento e manejo pesqueiro das espécies exploradas.

PALAVRAS-CHAVE. Pesca, manejo, siri, fauna-acompanhante.

Na Baía de Guanabara, Callinectes danae Smith, 1869 e C. ornatus Ordway, 1863 são duas espécies simpátricas e sintópicas, sendo amplamente distribuídas no Atlântico ocidental (Melo, 1996); constituem um recurso pesqueiro valioso, além de serem muito capturadas como fauna-acompanhante nas pescarias de arrasto de camarões realizadas no sudeste e sul do Brasil.

No Brasil, ambas espécies foram muito estudadas nas últimas duas décadas sob diversos aspectos (PITA et al., 1985; Branco \& MASUNARI, 1992; BRANCO \& LunARdon-Branco，1993; Negreiros-Fransozo \& Fransozo, 1995; Mantelatto \& Fransozo, 1996; Costa \& Negreiros-Fransozo, 1998; Negreiros-Fransozo et al., 1999; Mantelatto \& Fransozo, 1999; Branco \& Masunari, 2000; Baptista et al., 2003; Branco \& FracAsso, 2004; BAPTISTA-METRI et al., 2005).

$\mathrm{Na}$ Baía de Guanabara os portunídeos correspondem, pela sua abundância, a um dos grupos mais relevantes da fauna da região (LAVRADO et al., 2000), de forma que sua dinâmica de ocupação do espaço pode ser considerada como representativa do restante do megabentos. Contudo, estudos que abordam o crescimento de $C$. danae e $C$. ornatus são restritos aos trabalhos conduzidos no sul do Brasil por BRANCO \& Masunari (1992) e Branco \& LunARdOn-Branco (1993).
O estudo do crescimento é de extrema importância, pois a obtenção correta dos parâmetros biológicos do modelo de crescimento de BERTALANFFY (1938) pode fornecer, $a$ posteriori, estimativas das taxas de mortalidade, empregadas no ordenamento e manejo pesqueiro das espécies comerciais.

Objetiva-se neste trabalho descrever as curvas de crescimento de $C$. danae e $C$. ornatus, bem como estimar a longevidade destas.

\section{MATERIAL E MÉTODOS}

Os indivíduos foram coletados quinzenalmente na Baía de Guanabara $\left(22^{\circ} \mathrm{S}, 48^{\circ} \mathrm{W}\right)$ no período de novembro de 2003 a maio de 2005 utilizando-se um barco de arrasto de popa com portas, típico da frota artesanal da baía; a duração de cada coleta foi de uma hora. As coletas não foram pontuais, mudando constantemente ao longo do período amostral em função da quantidade de camarão e do conhecimento empírico do mestre da embarcação. $\mathrm{Na}$ maioria das vezes o sentido foi da ponte Rio-Niterói para o interior da baía, até próximo à ilha de Paquetá.

Os animais foram identificados em laboratório, sexados e medidos em sua maior largura, incluindo os espinhos laterais da carapaça, em milímetros. 
Para a análise do crescimento, os arrastos foram agrupados mensalmente e indivíduos separados em classes de $5 \mathrm{~mm}$ de largura. Posteriormente, foram obtidos modas ou picos modais onde as distribuições de frequiências de ocorrência observadas foram ajustadas a curvas normais.

A seleção das coortes para a construção das curvas de crescimento foi baseada na coerência dos parâmetros de BERTALANFFY (1938), calculados a partir da ferramenta Solver (Office XP). Para o L $\infty$ foi determinado um valor fixo, considerando o maior tamanho de machos e de fêmeas encontrados separadamente durante as coletas.

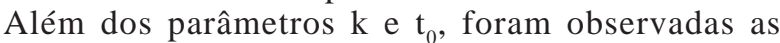
estimativas de longevidade e o $\mathrm{R}^{2}$ (acima de 0,9) de cada coorte. Ao final, somente aquelas coortes com variáveis coerentes com o ciclo de vida das espécies foram selecionadas. Uma vez selecionadas as coortes, procedeuse ainda o ajuste das idades (em dias) conforme o valor de $t_{0}$. Em uma última etapa, a união dos picos modais gerados e das respectivas idades corrigidas de todas as coortes selecionadas, originaram as curvas de crescimento: $\mathrm{LC}=\mathrm{L} \quad\left(1-\mathrm{e}^{-\mathrm{k}(\mathrm{t}-\mathrm{t} 0)}\right)$, onde LCoé a largura da carapaça no instante $t, L$ é a largura máxima quexem média a carapaça pode atingir (assíntota da curva), $\mathrm{k}$ é a constante de crescimento e $\mathrm{t}_{0}$ um parâmetro de ajuste que corresponde ao valor no eixo das abcissas (t), onde a curva intercepta, e está relacionado à largura da carapaça no instante do nascimento $(\mathrm{t}=$ zero $)$. Nesse caso, tanto para $C$. danae quanto para C. ornatus o tamanho da carapaça é desprezível ao nascer assumindo-se $\mathrm{t}_{0}$ igual a zero.

As curvas de machos e fêmeas foram comparadas empregando-se um teste F para ambas espécies, baseado em Cerrato (1990). A longevidade foi calculada pela fórmula inversa de von Bertalanffy modificada por D'INCAO \& FonseCA (1999), que consideram 99\% do tamanho assintótico.

\section{RESULTADOS}

Foram coletados 1.811 indivíduos de $C$. danae, sendo 1.442 fêmeas e 369 machos (Fig. 1), os maiores exemplares respectivamente com $113 \mathrm{~mm}$ e $120 \mathrm{~mm}$ de LC. C. ornatus totalizou 1.948 indivíduos, de onde foram observados 695 fêmeas e 1.253 machos (Fig. 2) e as maiores medidas de LC correspondentes foram $110 \mathrm{~mm}$ e $94 \mathrm{~mm}$. Em função da dispersão das modas calculadas, foram selecionadas cinco coortes para as fêmeas e sete para os machos de $C$. danae (Figs. 3,4); quatro coortes para as fêmeas e seis para os machos de $C$. ornatus (Figs. 5,6).

A comparação das curvas de crescimento, pelo teste $\mathrm{F}$ (Tab. I), demonstrou que uma única curva não descreve o crescimento de machos e fêmeas de $C$. danae (Figs. 7,8); contrariamente a C. ornatus (Figs. 9,10).

Tabela I. Teste F para comparação entre curvas de crescimento de machos e de fêmeas de Callinectes danae Smith, 1869 e Callinectes ornatus Ordway, 1863 obtidos no período de novembro de 2003 a maio de 2005 na Baía de Guanabara, Rio de Janeiro.

\begin{tabular}{lcc}
\hline & F calculado & F tabelado \\
\hline Callinectes danae & 18,64913 & 3,135918 \\
Callinectes ornatus & 0,418052 & 3,186582 \\
\hline
\end{tabular}

A longevidade alcançada para as espécies foi acima dos dois anos de idade. Estimou-se 2,3 e 2,5 anos para machos e fêmeas, respectivamente, de $C$. danae e 2,3 e 2,4 anos para machos e fêmeas de $C$. ornatus.

\section{DISCUSSÃO}

As curvas de crescimento de $C$. danae encontramse dentro de padrões esperados para crustáceos, em que um dos sexos tende a crescer mais do que o outro. As fêmeas costumam canalizar sua energia para a reprodução, desde a maturação gonadal até a liberação dos ovos, enquanto que os machos investem no crescimento. A diferença de tamanho entre os sexos de $C$. danae também poderia ser atribuída ao fato dos machos possuírem crescimento diferencial durante a puberdade, favorecendo o comportamento de proteção à fêmea no transcorrer do acasalamento (COSTA \& NegreirosFRANSOZO, 1998).

O padrão encontrado para $C$. ornatus revelou que apenas uma curva poderia ser aplicada para descrever o crescimento da espécie, independentemente do sexo; o esperado seria a obtenção de duas curvas de crescimento, uma para cada sexo. $\mathrm{O}$ crescimento e a distribuição de $C$. ornatus em ambientes tropicais e subtropicais ainda não foram detalhadamente investigados (NEGREIROS-FrANSOZO et. al., 1999). Entretanto, ao que tudo indica, C. ornatus apresenta um comportamento estratificado segundo o sexo e o tamanho de indivíduos (as fêmeas que parecem se concentrar em áreas mais rasas ou estuarinas quando jovens) (PITA et. al., 1985; BAPTISTA et. al., 2003). As águas com baixa salinidade são as mais apropriadas para berçários e estão associadas a um crescimento rápido e níveis de predação reduzidos, sendo vitais para a manutenção das populações locais (PosEY et al., 2005). Embora $C$. ornatus possa habitar mesmo a água doce (WILliams, 1974), possui uma associação significativa

Callinectes danae

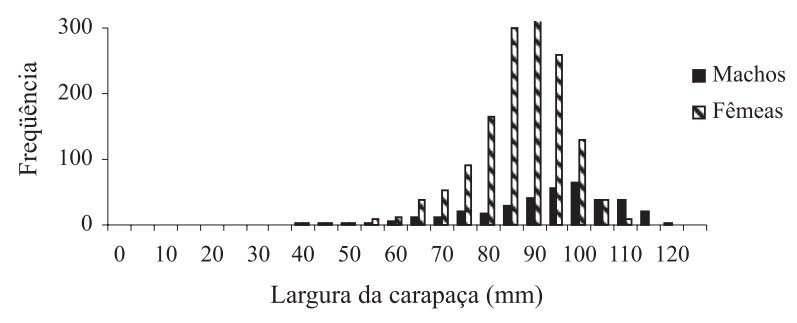

Callinectes ornatus

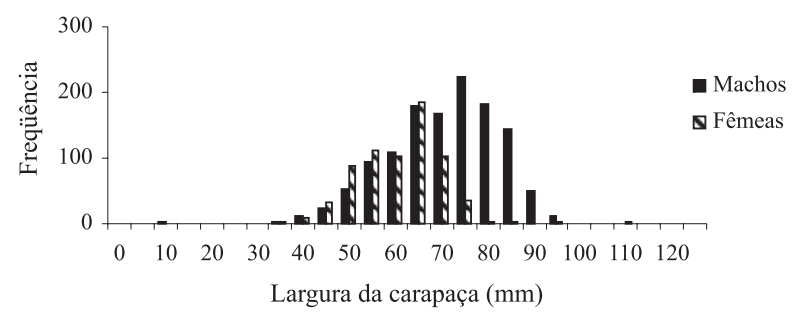

Figs. 1, 2. Distribuição das freqüências de ocorrência de machos e fêmeas de Callinectes danae Smith, 1869 e Callinectes ornatus Ordway, 1863 durante o período de novembro de 2003 a maio de 2005 na Baía de Guanabara, Rio de Janeiro. 
com a salinidade (NEgREIRos-Fransozo \& FrAnsozo, 1995), fator que desencadearia uma atividade migratória para águas mais salinas para completar o seu desenvolvimento e iniciar a desova. A migração já foi observada em indivíduos de Callinectes sapidus Rathbun, 1854, maiores que $24 \mathrm{~mm}$ de carapaça, que se deslocam para águas mais salinas para desovar (AGUILAR et al., 2005; Posey et al., 2005).
A distribuição de frequiências de $C$. ornatus na Baía de Guanabara apresenta uma estrutura populacional baseada em indivíduos jovens. $\mathrm{O}$ tamanho de primeira maturação gonadal das fêmeas de $C$. ornatus no Paraná foi estimado em $61 \mathrm{~mm}$ por BRANCO \& LUNARDON-BRANCO (1993). Através dessa estimativa, constatou-se que na Baía de Guanabara 56,6\% das fêmeas de C. ornatus estão abaixo do tamanho de primeira maturação gonadal. Essa
Callinectes danae

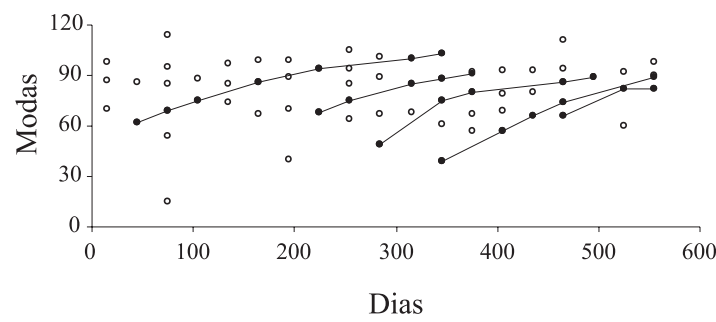

Callinectes danae

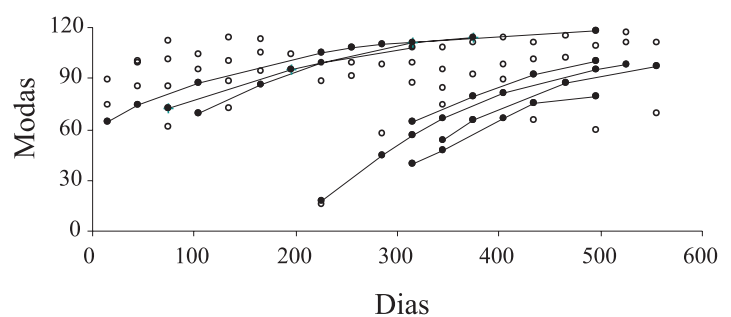

3

Callinectes ornatus

5

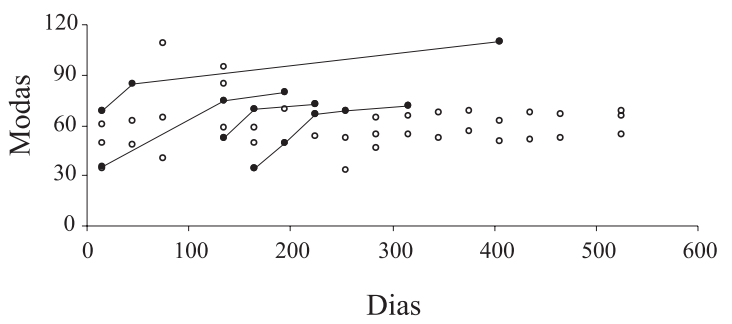

4

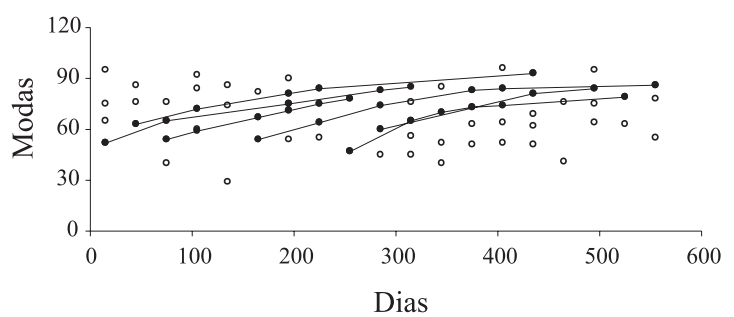

Figs. 3-6. Dispersão das modas e coortes selecionadas na Baía de Guanabara, Rio de Janeiro. Callinectes danae Smith, 1869: 3, fêmeas; 4, machos. Callinectes ornatus Ordway, 1863: 5, fêmeas; 6, machos.

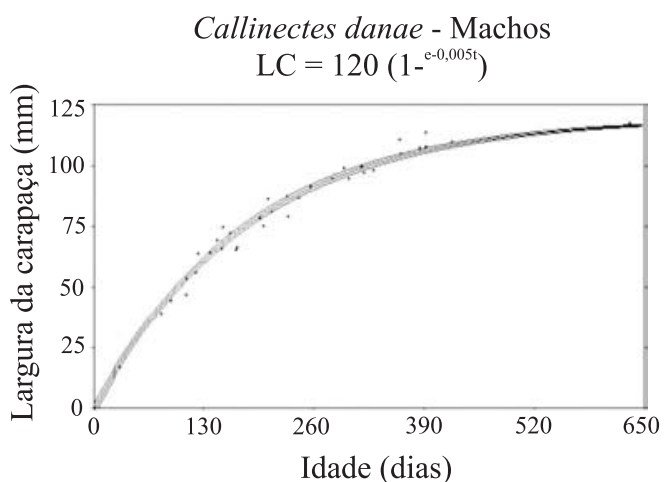

Callinectes danae - Fêmeas

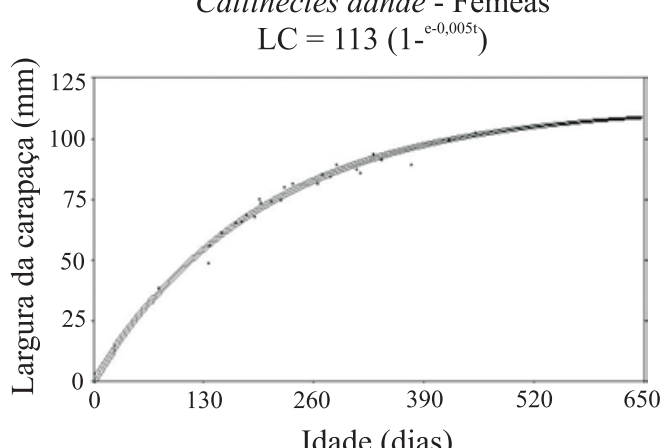

7

Callinectes ornatus - Machos

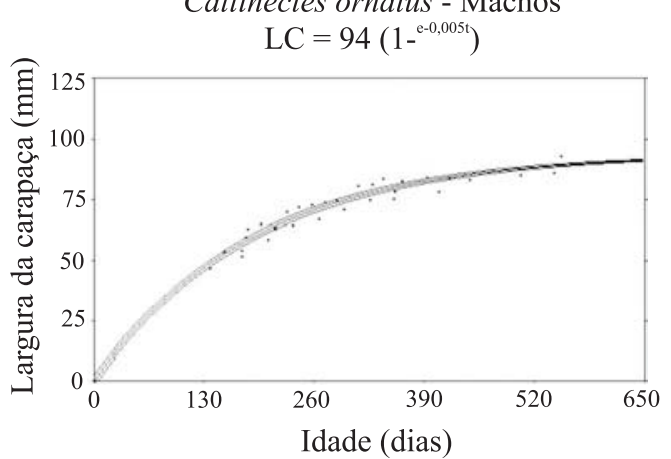

8

Callinectes ornatus - Fêmeas

10

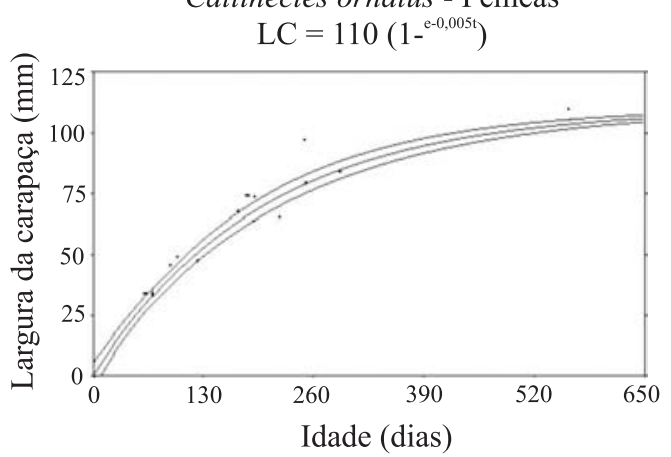

Figs. 7-10. Curva de crescimento obtida no período de novembro de 2003 a maio de 2005 na Baía de Guanabara, Rio de Janeiro (intervalo de confiança de 95\%). Callinectes danae Smith, 1869: 7, machos; 8, fêmeas; Callinectes ornatus Ordway, 1863: 9, machos; 10, fêmeas. 
predominância pode indicar que apenas uma curva descreveria o crescimento da população, como demonstrado pelo teste F. Entretanto, aceitando-se uma única curva para machos e fêmeas assume-se um erro, reflexo do elevado número de indivíduos jovens de $C$. ornatus na Baía de Guanabara, e também da ausência de fêmeas nas classes de maior LC, que emigrariam para áreas adjacentes à baía interferindo no resultado do teste aplicado.

Em relação às larguras assintóticas estimadas, somente constam na literatura as de BRANCO \& MASUNARI (1992) com C. danae e as de BRANCO \& LUNARDON-BRANCO (1993) com C. ornatus. As estimativas de ambas espécies concordam com as do presente trabalho. No entanto, a largura assintótica estimada por BRANCO \& MASUNARI (1992) para $C$. danae parece um pouco elevada, principalmente para fêmeas; contudo, não é possível afirmar que estejam superestimadas. Neste trabalho optou-se empregar as larguras máximas observadas por sexo e por espécie como a largura assintótica. Essa escolha foi baseada na possibilidade de crescimento diferencial entre os sexos e nas baixas abundâncias nas maiores classes de tamanho, principalmente das fêmeas de C. ornatus, que poderiam subestimar a largura assintótica.

Os valores estimados do parâmetro $\mathrm{k}$ para ambas espécies discordam daqueles encontrados por BRANCO \& MASUNARI (1992) e BRANCO \& LUNARDON-BRANCO (1993) (respectivamente 0,6975 e 0,6555 para machos e fêmeas de $C$. danae; 0,5160 e 0,6552 para machos e fêmeas de $C$. ornatus). Em bases anuais, os parâmetros $\mathrm{k}$ estimados para $C$. danae e $C$. ornatus da Baía de Guanabara (em torno de 2,0) foram superiores aos encontrados por esses autores. Contudo, as estimativas de $\mathrm{k}$ obtidas pelos autores acima são demasiadamente baixas quando comparadas com as apresentadas neste trabalho, o que pode ser reflexo da metodologia ou das variáveis abióticas atuando de forma diferenciada nos distintos sistemas. Em espécies simpátricas e sintópicas, com ciclos de vida semelhantes e sujeitas às mesmas variáveis ambientais, pode haver uma convergência dos valores de $\mathrm{k}$. Com efeito, no presente trabalho esses valores foram muito próximos, apresentando variações inter e intra-específicas muito pequenas, em torno de 0,005 dias. Tal hipótese também poderia ser usada para exlicar os baixos valores de k encontrados por BRANCO \& MASUNARI (1992) e BRANCO \& LUNARDON-BRANCO (1993) (em torno de 0,001 dias), como consequiência de uma relação direta do metabolismo com temperaturas menos elevadas da Região Sul do Brasil, ocasionando uma redução na taxa de crescimento das espécies.

Infelizmente a escassez de dados sobre o crescimento de representantes do gênero Callinectes não permite muitas comparações do parâmetro k. Ju et al. (2001) relataram que k em Callinectes sapidus pode variar de 0,51 a 1,45 ; entretanto, salientam que (na maioria dos casos) os parâmetros são obtidos a partir de experimentos de campo e de laboratório com animais cultivados. Em outras pesquisas com espécies de Brachyura (e.g. Chasmagnathus granulata, Portunus pelagicus, Armases rubripes) em ambientes naturais, o k variou de 1,60 a 2,55/ano (D'InCAO et al., 1993; JosiLEEN \& MENON, 2005; PIMENTA et al., 2005).
A possível subestimação do parâmetro k de $C$. ornatus e $C$. danae em Matinhos, Paraná e na Lagoa da Conceição, Santa Catarina (Branco \& MAsunari, 1992; Branco \& LunARdon-Branco, 1993) no entanto, pode ter causado a superestimação da longevidade para 3,0 e 3,5 anos, respectivamente. No presente trabalho, a longevidade verificada foi de no máximo 2,5 anos para as fêmeas de $C$. danae. Ainda acerca da longevidade, cabe destacar a metodologia empregada para estimá-la; D'INCAO \& FonseCA (1999) sugeriram validar a curva de crescimento por meio de estimadores de longevidade, assumindo que o comprimento assintótico (ou 99\% do $\mathrm{L}_{\infty}$ da curva de crescimento de Bertalanffy) tenham significado biológico, no entanto, os trabalhos de BRANCO \& MASUNARI (1992) e BRANCO \& LunARDON-BRANCO (1993) são anteriores ao de D' INCAO \& FonsECA (1999).

Por fim, a subestimação do parâmetro k não refletiria apenas valores equivocados de longevidade, podendo gerar um efeito cascata de forma negativa aos coeficientes de mortalidade e levar a medidas ineficientes de manejo pesqueiro (principalmente tratando-se de espécies de importância comercial).

Agradecimentos. A CAPES, FAPERJ e FUJB (Fundação Universitária José Bonifácio) pelo apoio financeiro ao projeto; à tripulação do barco Dona Dulce, pelo apoio logístico durante o período de coletas.

\section{REFERÊNCIAS BIBLIOGRÁFICAS}

Aguilar, R.; Hines, A. H.; Wolcott, T. G.; Wolcott, D. L.; Kramer, M. A. \& Lipcius, R. N. 2005. The timing and route of movement and migration of post-copulatory female blue crabs, Callinectes sapidus Rathbun, from the upper Chesapeake Bay. Journal of Experimental Marine Biology and Ecology 319:117-128.

Baptista, C.; Pinheiro, M. A. A.; Blankensteyn, A.; \& Borzone, C. A. 2003. Estrutura populacional de Callinectes ornatus Ordway (Crustacea, Portunidae) no Balneário Shangri-lá, Pontal do Paraná, Paraná, Brasil. Revista Brasileira de Zoologia 20(4):661-664.

Baptista-metri, C.; Pinheiro, M. A. A.; Blankensteyn, A.; \& Borzone, C. A. 2005. Biologia populacional de Callinectes danae Smith (Crustacea, Portunidae) no Balneário Shangri-lá, Pontal do Paraná, Paraná, Brasil. Revista Brasileira de Zoologia 22(2):446-453.

BertalanfFy, L. von. 1938. A quantitative theory of organic growth (Inquitiesom growth laws II). Human Biology 10(2): 181-213.

Branco, J. O. \& Fracasso, H. 2004. Biologia populacional de Callinectes ornatus (Ordway) na Armação do Itapocoroy, Penha, Santa Catarina, Brasil. Revista Brasileira de Zoologia 21(1):91-96.

Branco, J. O. \& Lunardon-Branco, M. J. 1993. Crescimento e tamanho de primeira maturação em Callinectes ornatus Ordway, 1863 (Decapoda, Portunidae) da região de Matinhos, Paraná, Brasil. Arquivos de Biologia e Tecnologia 36(3):497-503.

Branco, J. O \& Masunari, S. 1992. Crescimento de Callinectes danae Smith (Decapoda, Portunidae) da Lagoa da Conceição, Florianópolis, Santa Catarina, Brasil. Revista Brasileira de Zoologia 9(1/2):53-66.

2000. Reproductive ecology of the blue crab Callinectes danae Smith, 1869 in the Conceição Lagoon system, Santa Catarina Isle, Brazil. Revista Brasileira de Zoologia 60(1): 17-27.

Cerrato, R. M. 1990. Interpretable statistical tests for growth comparisons using parameters in the von Bertalanffy equation. Canadian Journal of Fisheries and Aquatic Sciences 47:1416-1426. 
Costa, M. C. \& Negreiros-Fransozo, M. L. 1998. The reproductive cycle of Callinectes danae Smith, 1869 (Decapoda, Portunidae) in the Ubatuba region, Brazil. Crustaceana 71(6):615-627.

D'Incao, F. \& FonsecA, D. B. 1999. The performance of the von Bertalanffy growth curve in penaeid shrimps: a critical aproache. In: Proceedings of the Fourth International Crustacean Congress. Amsterdam, The Netherlands. p. $733-737$.

D'Incao, F.; Ruffino, M. L.; Silva, K. G.; Braga, A. C. \& Marques, L. H. C. 1993. Crescimento de Chasmagnathus granulata Dana, 1851, em um marisma do estuário da Lagoa dos Patos, RS (Decapoda: Grapsidae). Revista Brasileira de Biologia 53:637-643

Josileen, J. \& Menon, N. G. 2005. Growth of the blue swimmer crab, Portunus pelagicus (Linnaeus, 1758) (Decapoda: Brachyura) in captivity. Crustaceana 78(1):1-18.

Ju, S. J.; Secor, D. H. \& Harvey, H. R. 2001. Growth rate variability and lipofuscin accumulation rates in the blue crab Callinectes sapidus. Marine Ecology Progress Series 224:107-205.

Lavrado, H. P.; Falcão, A. P. C.; Carvalho-Cunha, P. \& Silva, S. H. G. 2000. Composition and distribution of Decapoda from Guanabara Bay, RJ. Nauplius 8(1):15-23.

Mantelatto, F. L. M. \& Fransozo, A. 1996. Size at sexual maturity in Callinectes ornatus (Brachyura, Portunidae) from the Ubatuba Region (SP), Brazil. Nauplius 4:29-38.

. 1999. Reproductive biology and moulting cycle of the crab
Callinectes ornatus (Decapoda, Portunidae) from the Ubatuba region, São Paulo, Brazil. Crustaceana 72(1):63-76.

Melo, G. A. S. 1996. Manual de Identificação dos Brachyura (Caranguejos e Siris) do Litoral Brasileiro. São Paulo, Plêiade/FAPESP. 604p.

Negreiros-Fransozo, M. L. \& Fransozo, A. 1995. On the distribution of Callinectes ornatus Ordway, 1863 and Callinectes danae Smith, 1869 (Brachyura, Portunidae) in the Fortaleza Bay, Ubatuba, Brazil. Iheringia, Série Zoologia, 79:13-25.

Negreiros-Fransozo, M. L.; Mantelatto, F. L. M. \& Fransozo, A. 1999. Population biology of Callinectes ornatus Ordway, 1863 (Decapoda, Portunidae) from Ubatuba (SP), Brazil. Scientia Marina 63(2): $157-163$.

Pimenta, A. M.; Barutot, R. A.; D’Incao, F. \& Fonseca, D. B. 2005. Growth of Armases rubripes (Rathbun, 1897) in the estuary of the Lagoa dos Patos, Southern Brazil. Nauplius 13(2): 183-189.

Pita, J. B.; Rodrigues, E. S.; Graça Lopes, R. \& Coelho, J. A. P. 1985. Levantamento da família Portunidae (Crustacea, Decapoda, Brachyura) no complexo Baía-Estuário de Santos, São Paulo, Brasil. Boletim do Instituto de Pesca 12(3):153-162.

Posey, M. H.; Alphin, T. D.; Harwell, H. \& Allen, B. 2005. Importance of low salinity areas for juvenile blue crabs, Callinectes sapidus Rathbun, in the river-dominated estuaries of southeastern United States. Journal of Experimental Marine Biology and Ecology 319:81-100.

Williams, A. B. 1974. The swimming crabs of the genus Callinectes (Decapoda: Portunidae). Fishery Bulletin 72(3):685-798.

Recebido em dezembro de 2006. Aceito em outubro de 2007. ISSN 0073-4721

Artigo disponível em: www.scielo.br/isz 